\title{
Hardy inequality and Pohozaev identity for operators with boundary singularities: some applications
}

\author{
L'inégalité de Hardy et l'identité de Pohozaev pour opérateurs \\ avec des singularités sur la frontière : quelques applications \\ Cristian Cazacu ${ }^{\mathrm{a}, \mathrm{b}}$, \\ ${ }^{a}$ BCAM - Basque Center for Applied Mathematics, Bizkaia Technology Park, Building 500, 48160 Derio, Spain \\ ${ }^{\mathrm{b}}$ Departamento de Matemáticas, Universidad Autónoma, 28049 Madrid, Spain
}

\begin{abstract}
We consider the Schrödinger operator $A_{\lambda}:=-\Delta-\lambda /|x|^{2}, \lambda \in \mathbb{R}$, when the singularity is located on the boundary of a smooth domain $\Omega \subset \mathbb{R}^{N}, N \geq 1$

The aim of this Note is two folded. Firstly, we justify the extension of the classical Pohozaev identity for the Laplacian to this case. The problem we address is very much related to Hardy-Poincaré inequalities with boundary singularities. Secondly, the new Pohozaev identity allows to develop the multiplier method for the wave and the Schrödinger equations. In this way we extend to the case of boundary singularities well known observability and control properties for the classical wave and Schrödinger equations when the singularity is placed in the interior of the domain (Vanconstenoble and Zuazua [17]).
\end{abstract}

\section{Résumé}

Nous allons considérer l'operateur de Schrödinger $A_{\lambda}:=-\Delta-\lambda /|x|^{2}, \lambda \in \mathbb{R}$, lorsque l'origine est située sur la frontiére d'un domaine borné et régulière $\Omega \subset \mathbb{R}^{N}, N \geq 1$.

Cette Note a deux objectifs. Premièrement, nous montrons l'extension de l'identité classique de Pohozaev pour le Laplacien dans ce cas. Le problème que nous abordons est très lié aux inégalités de Hardy-Poincaré avec des singularités sur la frontiére. En second lieu, la nouvelle identité de Pohozaev permet de dériver le methode de multiplicateurs pour les équations des ondes et de Schrödinger. De cette façon, nous étendons au cas de la singularité frontalière propriétés d'observabilité et contrôle pour les équations des ondes classique et de Schrödinger bien connues dans le cas d'une singularité à l'interieur (Vancostenoble et Zuazua [17])

\section{Version française abrégée}

Email address: cazacu@bcamath.org (Cristian Cazacu). 
Dans cette Note nous nous intéressons à l'opérateur $A_{\lambda}:=-\Delta-\lambda /|x|^{2}, \lambda \in \mathbb{R}$, lorsque l'origine est située sur la frontière d'un domaine régulier $\Omega \subset \mathbb{R}^{N}, N \geq 1$. Il est connu que la valeur $\lambda(N):=$ $N^{2} / 4$, qui est la meilleure constante dans les inégalités de Hardy ci-après, est critique lorsque l'on étudie les propriétés qualitatives de $A_{\lambda}$. Dans la première partie de cette Note nous montrons que pour tout $\lambda \leq \lambda(N)$, l'identité de Pohozaev (voir Théorème 2) est vérifiée dans le domaine de $A_{\lambda}$, défini par $D\left(A_{\lambda}\right):=\left\{u \in H_{\lambda} \mid A_{\lambda} u \in L^{2}(\Omega)\right\}$. Nous allons définir plus tard l'espace $H_{\lambda}$ et quelques unes de ses propriétés. Formellement, le Théorème 2 peut être obtenu par intégration directe. Cependant, la singularité $x=0$ engendre une perte de régularité de l'opérateur $\left(A_{\lambda}, D\left(A_{\lambda}\right)\right)$ et les intégrations par parties ne sont plus justifiées rigoureusement. De plus, la régularité $L^{2}$ de la dérivée normale n'a plus lieu car les estimations elliptiques standards ne s'appliquent plus puisque la singularité est localisée sur le bord. Néanmoins, la trace d'un élément de $D\left(A_{\lambda}\right)$ existe dans un espace $L^{2}$ à poids, dont le poids est généré à l'origine, comme il est montré dans le Théorème 1. Dans la deuxième partie de cette Note, nous montrons plusieurs applications des Théorèmes 1, 2. D'abord des solutions non-triviales d'une EDP singulière sont traitées dans le Théorème 3 . Ensuite, nous dérivons des techniques de multiplicateurs afin de prouver "la régularité cachée" de la dérivée normale dans le cas de l'équation des ondes et de l'équation de Schrödinger, correspondant à $A_{\lambda}$ (voir le Théorème 4). En particulier, nous répondons à la question concernant la controlabilité des systèmes conservatifs. Le résultat principal est donné par le Théorème 5 et est dû à l'identité des multiplicateurs (10), en combinaison avec une inégalité forte de Hardy, formulée dans le Théorème 6 . Pour plus de clareté dand la présentation, nous allons traiter notamment le cas $\mathrm{C} 1$ de la figure 1. Cependant, les mêmes résultats peuvent être étendus aux cas C2, C3, C4 dans un cadre fonctionnel plus faible, dû à l'inégalité plus faible de Hardy (2).

\section{Introduction}

Let us consider $\Omega$ to be a smooth subset of $\mathbb{R}^{N}, N \geq 1$, with the origin $x=0$ placed on its boundary $\Gamma$. Without losing the generality we distinguish the following geometrical configurations for $\Omega$ as in Figure 1: C1 - $\Omega$ is a subset of $\mathbb{R}_{+}^{N}:=\left\{x=\left(x_{1}, \ldots, x_{N}\right) \in \mathbb{R}^{N} \mid x_{N}>0\right\}$ (Fig. 1, top left). C2 - Close to $x=0$, the points $x \in \Gamma$ satisfy $x \cdot \nu \geq 0$. Nevertheless, $\Omega$ crosses the hyperplane $x_{N}=0$ far from origin (Fig. 1 , top, right). C3 - Close to $x=0$, the points $x \in \Gamma$ verify $x \cdot \nu \leq 0$ (Fig. 1, bottom, left). C4 - For $x \in \Gamma$ the sign of $x \cdot \nu$ changes at origin (Fig. 1, bottom, right).

The following Hardy inequalities are well-known: if $\Omega$ verifies the case C1 in Fig. 1, then (e.g. [4]) for any $u \in C_{0}^{\infty}(\Omega)$ it holds that

$$
\int_{\Omega}|\nabla u|^{2} d x \geq \frac{N^{2}}{4} \int_{\Omega} \frac{u^{2}}{|x|^{2}} d x+\frac{1}{4} \int_{\Omega} \frac{u^{2}}{|x|^{2} \log ^{2}\left(R_{\Omega} /|x|\right)} d x .
$$

where $R_{\Omega}=\sup _{x \in \bar{\Omega}}|x|$. If $\Omega$ satisfies the cases C2, C3, C4 as in Fig. 1 then (e.g. [8], [9]) there exist $C_{2}=C_{2}(\Omega) \in \mathbb{R}$ and $C_{3}=C_{3}(\Omega, N)>0$ such that any $u \in C_{0}^{\infty}(\Omega)$ satisfies

$$
C_{2} \int_{\Omega} u^{2} d x+\int_{\Omega}|\nabla u|^{2} d x \geq \frac{N^{2}}{4} \int_{\Omega} \frac{u^{2}}{|x|^{2}} d x+C_{3} \int_{\Omega} \frac{u^{2}}{|x|^{2} \log ^{2}\left(R_{\Omega} /|x|\right)} d x .
$$

In both situations above, the constant $\lambda(N)=N^{2} / 4$ is optimal. For the sake of clarity and because similar results can be obtained in the other cases in an other functional framework, next we analyze the situation when $\Omega$ verifies the case $\mathrm{C} 1$ in Fig. 1 . In the sequel let us consider the operator $\left(A_{\lambda}, D\left(A_{\lambda}\right)\right)$, $\lambda \leq \lambda(N)$, acting on such $\Omega$.

Firstly, this Note is aimed to justify the Pohozaev identity $(5)$ in the functional setting $\left(A_{\lambda}, D\left(A_{\lambda}\right)\right)$ in which Theorem 1 plays a crucial role. Pohozaev-type identities (e.g. pp. 515, [7]) have been widely used to show non-existence results to nonlinear elliptic equations. In particular, we point out that this issue has been also studied for nonlinear equations with singular potentials (see e.g. [5], [10]). In those cases, due to the regularizing effect of the nonlinearity, the solutions become regular enough to obtain the corresponding Pohozaev identity by direct computations. This is not precisely our case. To the best of 
C1: The elliptic case

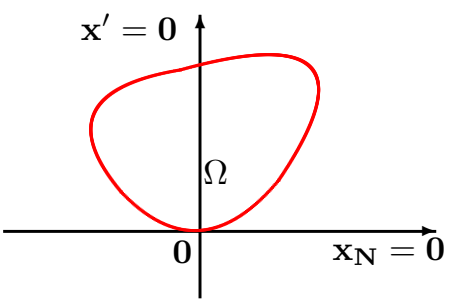

C3: The hyperbolic case

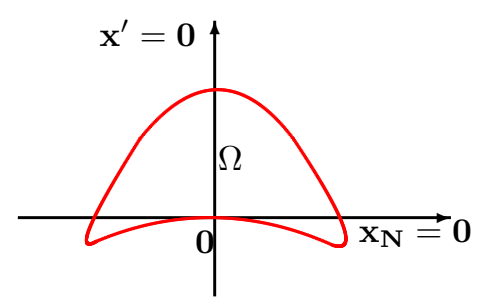

C2: The locally elliptic case

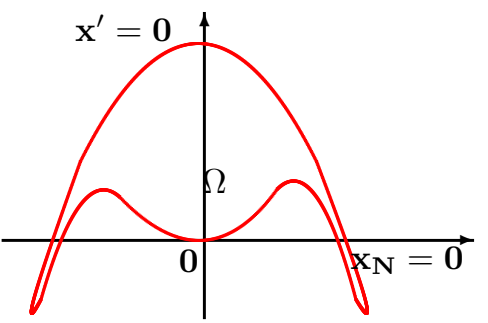

C4: $x \cdot \nu$ changes sign at 0

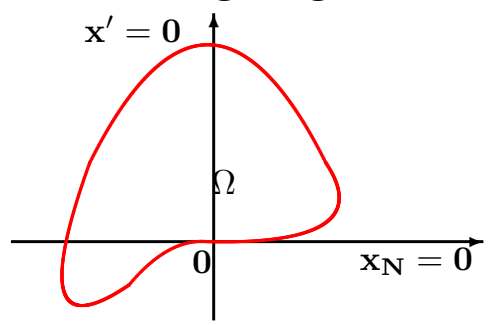

Figure 1. Geometry of $\Omega$ (in red), $\nu$ denotes the outward normal vector

our knowledge, the regularity of the operator $\left(A_{\lambda}, D\left(A_{\lambda}\right)\right)$ in terms of the Sobolev spaces is yet unknown and this affects the direct justification of (5). To do this we proceed by approximations arguments near the singularity. We analyze two situations when discussing the values of $\lambda$ : the subcritical case $\lambda \leq \lambda(N)$ respectively the critical case $\lambda=\lambda(N)$. However, the main novelty appears for the critical value $\lambda=\lambda(N)$ in which case $H_{\lambda}$ is strictly larger than $H_{0}^{1}(\Omega)$. This case requires e better understanding of the norm of $H_{\lambda(N)}$ as discussed in Section 2.

The controllability properties and stabilization of the heat and wave equation corresponding to $A_{\lambda}$ have been analyzed in [16], [6], [17] in the case of interior singularity. Roughly speaking, they showed that the parameter $\lambda_{\star}=(N-2)^{2} / 4$, which is the optimal constant in the Hardy inequality with interior singularity, is critical when asking the well-posedness and control properties of such systems. In the second part of this Note we address the question of controllability for the wave and Schrodinger equations corresponding to $A_{\lambda}$, in the case of boundary singularity. Our main result asserts that we can increase the range of values $\lambda$ (from $\lambda_{\star}$ to $\lambda(N)$ ) for which the exact controllability holds.

2. The space $H_{\lambda}$, proper norm and main elliptic results. Following the idea in [18], thanks to inequality (1) we consider the Hardy functional $B_{\lambda}[u]=\int_{\Omega}\left[|\nabla u|^{2}-\lambda u^{2} /|x|^{2}\right] d x$, which is positive and finite for all $u \in C_{0}^{\infty}(\Omega)$. We define the Hilbert space $H_{\lambda}$ to be the completion of $C_{0}^{\infty}(\Omega)$ functions in the norm induced by $B_{\lambda}[u]$. If $\lambda<\lambda(N)$, it holds that $H_{0}^{1}(\Omega)=H_{\lambda}$ due to Hardy inequality which ensures the equivalence of the norms. Similar to the case of interior singularity emphasized in [19], an interesting phenomena appears in the critical space $H_{\lambda(N)}$. Assume $\Omega=\left\{x \in \mathbb{R}^{N}|| x \mid \leq 1, x_{N}>\right.$ $0\}$ and let $e_{1}:=x_{N}|x|^{-N / 2} J\left(z_{0,1}|x|\right)$ where $z_{0,1}$ is the first positive zero of the Bessel function $J_{0}$. Then there exists $\lim _{\epsilon \rightarrow 0} \int_{x \in \Omega,|x| \geq \epsilon}\left[\left|\nabla e_{1}\right|^{2}-\lambda(N) e_{1}^{2}|x|^{2}\right] d x<\infty$ although $e_{1} \notin H_{0}^{1}(\Omega)$. Surprisingly, the meaning of $B_{\lambda(N)}[\cdot]$ is not well-defined in the sense of principle value. Indeed, if it were one can check that $B_{\lambda(N)}\left[e_{1}-\phi\right] \geq C_{0}>0$, for all $\phi \in C_{0}^{\infty}(\Omega)$ for some universal constant $C_{0}>0$. This is in contradiction with the definition of $H_{\lambda(N)}$ ! The remedy for this is to consider the functional 


$$
B_{\lambda, 1}[u]=\int_{\Omega}\left|\nabla u+\frac{N}{2} \frac{x}{|x|^{2}} u-\frac{e_{N}}{x_{N}} u\right|^{2} d x+(\lambda(N)-\lambda) \int_{\Omega} \frac{u^{2}}{|x|^{2}} d x,
$$

where $e_{N}$ denotes the $N$-th canonical vector of $\mathbb{R}^{N}$. We observe that $B_{\lambda}[u]=B_{\lambda, 1}[u]$, for all $u \in C_{0}^{\infty}(\Omega)$. Therefore, $B_{\lambda, 1}[u]$ induces a new norm in the space $H_{\lambda}$ which is well understood in the sense of principal value. In the sequel, we denote by $\|\cdot\|_{H_{\lambda}}$ the norm induced by $B_{\lambda, 1}$.

Notations: For any $\epsilon>0, \theta_{\epsilon}$ is a smooth cut-off function which satisfies $\theta_{\epsilon}=0$ for $|x| \leq \epsilon$ respectively $\theta_{\epsilon}=1$ for $|x| \geq 2 \epsilon$. Besides, $\boldsymbol{q} \in\left(C^{2}(\bar{\Omega})\right)^{N}$ denotes a vector field such that $\boldsymbol{q}=\nu$ on $\Gamma$. $H_{\lambda}^{\prime}$ denotes the dual space of $H_{\lambda}$. Next we state our main elliptic results.

Theorem 1 (trace regularity) Assume $\Omega \subset \mathbb{R}^{N}, N \geq 1$, verifies the case C1. Let us consider $\lambda \leq$ $\lambda(N)$ and $u \in D\left(A_{\lambda}\right)$. Then $(\partial u / \partial \nu)|x| \in L^{2}(\Gamma)$ and there exists $C=C(\Omega)>0$ such that

$$
\int_{\Gamma}\left(\frac{\partial u}{\partial \nu}\right)^{2}|x|^{2} d \sigma \leq C\left(\|u\|_{H_{\lambda}}^{2}+\left\|A_{\lambda} u\right\|_{L^{2}(\Omega)}^{2}\right) .
$$

Sketch of the proof. In order to avoid the singularity, we multiply $A_{\lambda} u$ by $|x|^{2}(\boldsymbol{q} \cdot \nabla u) \theta_{\epsilon}$ and we integrate by parts. Then we obtain an identity which, combined with Cauchy-Schwartz inequality, allows to get uniform upper bounds for the boundary term. Then, by Fatou Lemma we can pass to the limit as $\epsilon$ tends to zero to end up the proof.

Theorem 2 (Pohozaev identity) Assume $\Omega \subset \mathbb{R}^{N}, N \geq 1$, verifies the case $C 1$ and let $\lambda \leq \lambda(N)$. Then for all $u \in D\left(A_{\lambda}\right)$ it holds that

$$
\frac{1}{2} \int_{\Gamma}(x \cdot \nu)\left(\frac{\partial u}{\partial \nu}\right)^{2} d \sigma=-\int_{\Omega} A_{\lambda} u(x \cdot \nabla u) d x-\frac{N-2}{2}\|u\|_{H_{\lambda}}^{2} .
$$

Sketch of the proof. Note firstly that all terms in (5) are finite. Indeed, thanks to the fact that $x \cdot \nu=$ $O\left(|x|^{2}\right)$ and Theorem 1 we obtain the integrability of the boundary term. Moreover, $x \cdot \nabla u \in L^{2}(\Omega)$ as shown in Theorem 6. We proceed by multiplier technique. If $\lambda<\lambda(N)$ the multiplier $(x \cdot \nabla u) \theta_{\epsilon}$ is used to obtain (5) in the limit process. If $\lambda=\lambda(N)$ we apply the subcritical result for $(\lambda(N)-\delta)$ and we argue we can pass to the limit as $\delta$ tends to zero. Indeed, following approximation lemma which is proved in generality in [1], for a given $u \in D\left(A_{\lambda}\right)$, the solution $u_{\delta}$ of $A_{\lambda(N)-\delta} u_{\delta}=A_{\lambda(N)} u$, converges strongly to $u$ in $H_{\lambda(N)}$. Secondly, by comparison arguments we show the asymptotic behavior of $u_{\delta}, \nabla u_{\delta}$ when $A_{\lambda} u$ is smooth. This is done by constructing super solutions and using rescalling arguments in [3] to get rid of the singularity. A density argument together with Theorem 1 concludes the proof.

Theorem 3 Assume $\lambda \leq \lambda(N)$ and $\Omega \subset \mathbb{R}^{N}, N \geq 3$, satisfying the case C1. Let us consider the problem

$$
-\Delta u-\frac{\lambda}{|x|^{2}} u=|u|^{\alpha-1} u, \quad x \in \Omega ; \quad u=0, \quad x \in \Gamma,
$$

1). If $1<\alpha<(N+2) /(N-2)$, problem (6) has non trivial solutions in $H_{\lambda}$. Moreover, if $1<\alpha<$ $N /(N-2)$ it has non trivial solutions in $D\left(A_{\lambda}\right)$.

2). Assume $\Omega$ is a star-shaped domain (i.e. $x \cdot \nu \geq 0$, for all $x \in \Gamma$ ). If $\alpha \geq(N+2) /(N-2)$, problem (6) does not have non trivial solutions in $D\left(A_{\lambda}\right)$.

Sketch of the proof. The existence of non-trivial solutions reduces to finding minimizers for the problem $I=\inf _{\|u\|_{L^{\alpha+1}(\Omega)}=1}\|u\|_{H_{\lambda}}^{2}$ and is due to the compact embedding $H_{\lambda} \hookrightarrow L^{\alpha+1}(\Omega)$. The non-existence result is proved by combining Theorem 2 and unique continuation results as in [12].

\section{Applications to controllability}

Let us set $\Gamma_{0}:=\{x \in \Gamma \mid x \cdot \nu \geq 0\}$. a non-empty part of the boundary $\Gamma$. Next we consider the Wave-like process 


$$
\begin{cases}u_{t t}-\Delta u-\lambda \frac{u}{|x|^{2}}=0, & (t, x) \in Q_{T}, \\ u(t, x)=h(t, x) \chi_{\Gamma_{0}}, & (t, x) \in \Sigma_{T}, \\ \left(u(0, x), u_{t}(0, x)\right)=\left(u_{0}(x), u_{1}(x)\right), & x \in \Omega,\end{cases}
$$

where $Q_{T}=(0, T) \times \Omega, \Sigma_{T}=(0, T) \times \Gamma$ and $\chi_{\Gamma_{0}}$ denotes the characteristic function of $\Gamma_{0}$.

The solution of (7) is defined in week sense by the transposition method (J.L. Lions [13]). In this section we address the question of exact controllability of system (7), i.e. whether for any initial data $\left(u_{0}, u_{1}\right) \in L^{2}(\Omega) \times H_{\lambda}^{\prime}$ and any target $\left(\overline{u_{0}}, \overline{u_{1}}\right) \in L^{2}(\Omega) \times H_{\lambda}^{\prime}$, there exists a finite time $T>0$ and a control $h \in L^{2}\left((0, T) \times \Gamma_{0}\right)$ such that the solution of $(7)$ satisfies $\left(u_{t}(T, x), u(T, x)\right)=\left(\overline{u_{1}}(x), \overline{u_{0}}(x)\right)$ for all $x \in \Omega$. In view of the time-reversibility of the equation it is enough to consider the null-controllability problem, i.e. the case where the target $\left(\overline{u_{0}}, \overline{u_{1}}\right)=(0,0)$. By now classical Hilbert Uniqueness Method (HUM) (see J. L. Lions [13]) the null-controllability of system (7) is characterized through the adjoint system

$$
\begin{cases}v_{t t}-\Delta v-\lambda \frac{v}{|x|^{2}}=0, & (t, x) \in Q_{T}, \\ v(t, x)=0, & (t, x) \in \Sigma_{T}, \\ \left(v(0, x), v_{t}(0, x)\right)=\left(v_{0}(x), v_{1}(x)\right), & x \in \Omega\end{cases}
$$

The operator $\left(\mathcal{A}_{\lambda}, D\left(\mathcal{A}_{\lambda}\right)\right)$ defined by $\mathcal{A}_{\lambda}(v, w)=\left(w, \Delta v+\lambda|x|^{2} v\right)$ for all $(v, w) \in D\left(\mathcal{A}_{\lambda}\right):=D\left(A_{\lambda}\right) \times H_{\lambda}$ generates the wave semigroup in $H_{\lambda} \times L^{2}(\Omega)$. In view of that, the adjoint system is well-posed.

In the sequel, we justify some "hidden regularity" effect for the system (8) which may not be directly deduce from the semigroup regularity but from the equation itself.

Theorem 4 (Hidden regularity) Assume $\lambda \leq \lambda(N)$ and $v$ is the solution of (8) corresponding to the initial data $\left(v_{0}, v_{1}\right) \in H_{\lambda} \times L^{2}(\Omega)$. Then $v$ satisfies

$$
\int_{0}^{T} \int_{\Gamma}\left(\frac{\partial v}{\partial \nu}\right)^{2}|x|^{2} d \sigma d t \leq C\left(\left\|v_{0}\right\|_{H_{\lambda}}^{2}+\left\|v_{1}\right\|_{L^{2}(\Omega)}^{2}\right) .
$$

for some universal constant $C>0$. Moreover, $v$ verifies the identity

$$
\frac{1}{2} \int_{0}^{T} \int_{\Gamma}(x \cdot \nu)\left(\frac{\partial v}{\partial \nu}\right)^{2} d \sigma d t=\frac{T}{2}\left(\left\|v_{0}\right\|_{H_{\lambda}}^{2}+\left\|v_{1}\right\|_{L^{2}(\Omega)}^{2}\right)+\left.\int_{\Omega} v_{t}\left(x \cdot \nabla v+\frac{N-1}{2} v\right)\right|_{0} ^{T} d x
$$

Sketch of the proof. By density, tt suffices to prove Theorem 4 , for initial data $\left(v_{0}, v_{1}\right)$ in $D\left(\mathcal{A}_{\lambda}\right)$. For the proof of (9) we multiply $A_{\lambda} v$ by $|x|^{2}(\boldsymbol{q} \cdot \nabla v) \theta_{\epsilon}$ and integrate. The integration in time and the conservation of energy allow to obtain uniform bounds for the boundary term in the energy space. Then by Fatou Lemma we pass to the limit as $\epsilon$ tends to zero and the proof finishes.

For the proof of (10) we proceed straightforward from Theorem 2. Indeed, for a fixed time $t \in[0, T]$ we apply Theorem 2 for $A_{\lambda} v=-v_{t t}$. Then we integrate in time, and due to the equipartition of energy we can finish the proof.

Due to Theorem 4 the operator $\left(v_{0}, v_{1}\right) \mapsto\left(\int_{0}^{T} \int_{\Gamma_{0}}(x \cdot \nu)(\partial v / \partial \nu)^{2} d \sigma d t\right)^{1 / 2}$ is a linear continuous map in $H_{\lambda} \times L^{2}(\Omega)$. Let $\mathcal{H}$ be the completion of this norm in $H_{\lambda} \times L^{2}(\Omega)$. We consider the functional $J: \mathcal{H} \rightarrow \mathbb{R}$ defined by

$$
J\left(v_{0}, v_{1}\right)(v):=\frac{1}{2} \int_{0}^{T} \int_{\Gamma_{0}}(x \cdot \nu)\left(\frac{\partial v}{\partial \nu}\right)^{2} d \sigma d t-<u_{1}, v_{0}>_{H_{\lambda}^{\prime}, H_{\lambda}}+\left(u_{0}, v_{1}\right)_{L^{2}(\Omega), L^{2}(\Omega)},
$$

where $v$ is the solution of (8) corresponding to initial data $\left(v_{0}, v_{1}\right)$. Of course, $\left\langle\cdot, \cdot>_{H_{\lambda}^{\prime}, H_{\lambda}}\right.$ denotes the duality product. The control $h \in L^{2}\left((0, T) \times \Gamma_{0}\right)$ for $(7)$ could be chosen as $h=(x \cdot \nu) v_{\text {min }}$ where 
$v_{\text {min }}$ minimizes the functional $J$ on $\mathcal{H}$ among the solutions $v$ of (8) corresponding to the initial data $\left(u_{0}, u_{1}\right) \in H_{\lambda}^{\prime} \times L^{2}(\Omega)$ The existence of a minimizer of $J$ is assured by the coercivity of $J$ which is equivalent to the Observability Inequality for the adjoint system stated as follows.

Theorem 5 (Observability) For all $\lambda \leq \lambda(N)$, there exists a positive constant $D_{1}=D_{1}(\Omega, \lambda, T)>0$ such that for all $T \geq 2 R_{\Omega}$, and any initial data $\left(v_{0}, v_{1}\right) \in H_{\lambda} \times L^{2}(\Omega)$ the solution of (8) verifies

$$
\left\|v_{0}\right\|_{H_{\lambda}}^{2}+\left\|v_{1}\right\|_{L^{2}(\Omega)}^{2} \leq D_{1} \int_{0}^{T} \int_{\Gamma_{0}}(x \cdot \nu)\left(\frac{\partial v}{\partial \nu}\right)^{2} d \sigma d t .
$$

Sketch of the proof. The proof of Theorem 5 relies mainly on Theorem 4, combining compactness uniqueness argument (cf. [14]) and the sharp Hardy inequality stated in Theorem 6.

Theorem 6 Assume $\Omega$ satisfies one of the cases C1-C4. Then, there exists a constant $C=C(\Omega) \in \mathbb{R}$ such that

$$
\int_{\Omega}|x|^{2}|\nabla v|^{2} d x \leq R_{\Omega}^{2}\left[\int_{\Omega}|\nabla v|^{2} d x-\frac{N^{2}}{4} \int_{\Omega} \frac{v^{2}}{|x|^{2}} d x\right]+C \int_{\Omega} v^{2} d x \quad \forall v \in C_{0}^{\infty}(\Omega) .
$$

Remark 1 The proof of Theorem 6 is quite technical and we omit it here. The constant $R_{\Omega}^{2}$ which appears in inequality (13), helps to obtain the control time $T>T_{0}=2 R_{\Omega}$, which is sharp from the Geometric Control Condition considerations, see [2].

The results above guarantee the exact boundary controllability of (7). More precisely, we obtain

Theorem 7 (Controllability) Assume that $\Omega$ satisfies $C 1$ and $\lambda \leq \lambda(N)$. For any time $T>2 R_{\Omega}$, $\left(u_{0}, u_{1}\right) \in L^{2}(\Omega) \times H_{\lambda}^{\prime}$ and $\left(\overline{u_{0}}, \overline{u_{1}}\right) \in L^{2}(\Omega) \times H_{\lambda}^{\prime}$ there exists $h \in L^{2}\left((0, T) \times \Gamma_{0}\right)$ such that the solution of (7) satisfies $\left(u_{t}(T, x), u(T, x)\right)=\left(\overline{u_{1}}(x), \overline{u_{0}}(x)\right)$ for all $x \in \Omega$.

Schrödinger equation. In the above geometrical settings, we consider the Schrödinger equation

$$
\begin{cases}i u_{t}-\Delta u-\lambda \frac{u}{|x|^{2}}=0, & (t, x) \in Q_{T}, \\ u(t, x)=h(t, x) \chi_{\Gamma_{0}}, & (t, x) \in \Sigma_{T}, \\ u(0, x)=u_{0}(x), & x \in \Omega,\end{cases}
$$

For all $\lambda \leq \lambda(N)$, we define the Hilbert space $H_{\lambda}(\Omega ; \mathbb{C})$ as the completion of $H_{0}^{1}(\Omega ; \mathbb{C})$ with respect to the norm induced by the inner product $\left\langle u, v>_{H_{\lambda}(\Omega ; \mathbb{C})}:=\operatorname{Re} \int_{\Omega}\left(\nabla u(x) \cdot \nabla \overline{v(x)}-\lambda u(x) \overline{v(x)} /|x|^{2}\right) d x\right.$. Then

Theorem 8 (Controllability) For any $\lambda \leq \lambda(N), u_{0} \in H_{\lambda}^{\prime}, \overline{u_{0}} \in H_{\lambda}^{\prime}$ and any time $T>0$ there exists $h \in L^{2}\left((0, T) \times \Gamma_{0}\right)$ such that the solution of $(14)$ satisfies $\left.u(T, x)\right)=\overline{u_{0}}(x)$ for all $x \in \Omega$.

This result holds true due to the result valid for the wave equation. Indeed, the general theory presented in an abstract form in [15], assure the observability of systems like $\dot{z}=i A_{0} z$ using results available for systems of the form $\ddot{z}=-A_{0} z$.

Acknowledgements. The author thanks Enrique Zuazua and Adi Adimurthi for fruitful discussions and suggestions.

Partially supported by the Grant MTM2008-03541 of the MICINN (Spain), project PI2010-04 of the Basque Government, the ERC Advanced Grant FP7-246775 NUMERIWAVES, the ESF Research Networking Program OPTPDE, the grant PN-II-ID-PCE-2011-3-0075 of CNCS-UEFISCDI Romania and a doctoral fellowship from UAM (Universidad Autónoma de Madrid).

\section{References}

[1] A. Adimurthi, C. Cazacu and E. Zuazua, Best constants and Pohozaev identity for Hardy-Sobolev type operators, in preparation. 
[2] C. Bardos, G. Lebeau and J. Rauch, Control and stabilization for hyperbolic equations, Mathematical and numerical aspects of wave propagation phenomena (Strasbourg, 1991), 252-266, SIAM, Philadelphia, PA, 1991.

[3] H. Brezis, M. Marcus and I. Shafrir, Extremal functions for Hardy's inequality with weight, J. Funct. Anal., 171, 2000, 1, 177-191.

[4] C. Cazacu, On Hardy inequalities with singularities on the boundary, C. R. Acad. Sci. Paris, Ser. I, 349, 2011, 273-277,

[5] J. Davila and I. Peral, Nonlinear elliptic problems with a singular weight on the boundary, Calc. Var. doi 10.1007/s00526010-0376-5.

[6] S. Ervedoza, Control and stabilization properties for a singular heat equation with an inverse-square potential, Comm. Partial Differential Equations, 33, 2008, 10-12, 1996-2019,

[7] L. C. Evans, Partial differential equations, Graduate Studies in Mathematics, 19, American Mathematical Society, Providence, RI, 2010, xxii+749.

[8] M. M. Fall, On the Hardy Poincaré inequality with boundary singularities. Commun. Contemp. Math, to appear.

[9] M. M. Fall and R. Musina, Hardy-Poincaré inequality with boundary singularities. Proc. Roy. Soc. Edinburgh, to appear.

[10] N. Ghoussoub, and C. Yuan, Multiple solutions for quasi-linear PDEs involving the critical Sobolev and Hardy exponents, Trans. Amer. Math. Soc., 352, 2000, 12, 5703-5743.

[11] G. H. Hardy, J. E. Littlewood, and G. Pólya, Inequalities, Cambridge Mathematical Library, Cambridge University Press, Cambridge, 1988, Reprint of the 1952 edition.

[12] D. Jerison and C. E. Kenig, Unique continuation and absence of positive eigenvalues for Schrödinger operators, with an appendix by E. M. Stein, Ann. of Math. (2), 121, 1985, 3, 463-494,

[13] J.-L. Lions, Contrôlabilité exacte, perturbations et stabilisation de systèmes distribués. Tome 1, Recherches en Mathématiques Appliquées [Research in Applied Mathematics], 8, Contrôlabilité exacte. [Exact controllability], With appendices by E. Zuazua, C. Bardos, G. Lebeau and J. Rauch, Masson, Paris, 1988,

[14] E. Machtyngier, Exact controllability for the Schrödinger equation, SIAM J. Control Optim., 32, 1994, 1, 24-34,

[15] M. Tucsnak and G. Weiss, Observation and control for operator semigroups, Birkhäuser Advanced Texts: Basler Lehrbücher. [Birkhäuser Advanced Texts: Basel Textbooks], Birkhäuser Verlag, Basel, 2009, xii+483.

[16] , J. Vancostenoble, J. and E. Zuazua, Null controllability for the heat equation with singular inverse-square potentials, J. Funct. Anal., 254, (2008) (7), 1864-1902.

[17] J. Vancostenoble and E. Zuazua, Hardy inequalities, observability, and control for the wave and Schrödinger equations with singular potentials, SIAM J. Math. Anal., 41, 2009, 4, 1508-1532.

[18] J. L. Vázquez and E. Zuazua, The Hardy inequality and the asymptotic behaviour of the heat equation with an inverse-square potential, J. Funct. Anal. 173 (2000), no. 1, 103-153.

[19] J. L. Vázquez and N. B. Zographopoulos, Functional aspects of the Hardy inequality. Appearance of a hidden energy, http://arxiv.org/abs/1102.5661. 